City University of New York (CUNY) CUNY Academic Works

1966

\title{
A Linear Model Approach to Time and Cost Analysis
}

William (Bill) H. Williams

CUNY Hunter College

\section{How does access to this work benefit you? Let us know!}

More information about this work at: https://academicworks.cuny.edu/hc_pubs/663

Discover additional works at: https://academicworks.cuny.edu

This work is made publicly available by the City University of New York (CUNY).

Contact: AcademicWorks@cuny.edu 


\title{
A LINEAR MODEL APPROACH TO TIME AND COST ANALYSIS*
}

\author{
W. H. WILLIAMS
}

\author{
Bell Telephone Laboratories, Inc., Murray Hill, New Jersey
}

\begin{abstract}
Systems analyses frequently require estimates of the average time that it takes to perform repetitive tasks. These time estimates may be easy to obtain; for example, if one man is performing a single operation, then a simple division of his total working time by the frequency of the task repetition, gives an estimate of the average performance time. However, in many instances, work consolidation requires that individuals be called upon to perform several different operations. For example, crews may be sent into the field to carry out a number of different tasks, for which the relative rate of occurrence may change from day to day. This introduction of multiple operations implies that the required separate time estimates can no longer be obtained by a simple division.

This paper discusses linear model estimation of the average time taken to perform specific tasks; the estimation is possible even when a number of different tasks are performed by an individual or group. The models are based on the conceptual relation that the time taken to perform a task multiplied by the rate of occurrence in the considered time period, and summed over all possible tasks, should equal the total time taken by all tasks. Estimation is also possible when direct observations are difficult to obtain (as in stopwatch procedures) because the proposed models do not require direct time observations but rather utilize linear combinations of the individual time parameters.

An actual application is discussed.
\end{abstract}

\section{Introduction}

\section{(a) Time Studies}

Systems analyses frequently require estimates of the average time that it takes to perform repetitive tasks. These time estimates may be easy to obtain; for example, if one man is performing a single operation, then a simple division of his total working time by the frequency of the task repetition, gives an estimate of the average performance time. However, in many instances, work consolidation requires that individuals be called upon to perform several different operations. For example, crews may be sent into the field to carry out a number of different tasks, the relative frequency of which may change from day to day. This introduction of multiple operations implies that the required separate time estimates can no longer be obtained by a simple division.

Time study analysis has a large literature, see, for example Abruzzi [1] and Krick [2]; and while it is not possible to review it here some comments on the classical techniques of stopwatch and time-slice work sampling will be helpful. The stopwatch procedure measures the time required to perform an operation

* Received May 1965; revised October 1965. 
once and repeated measurements of the same operation lead to estimates with a smaller variance. Unfortunately, these estimates can be badly biased by the very act of observation; and efforts to get around this potential bias have led to the recent increase in the popularity of time-slice work sampling. This latter method requires observation of the specific task of an individual at randomly selected times of the day and it claims to avoid the bias of the stopwatch procedure. Both of these are familiar techniques; but it should be emphasized, that, in its simplest form, the time-slice procedure estimates the proportion of time spent on a certain type of task and not the average time per occurrence. The latter can be obtained from time-slice data by concurrently observing the frequency of repetition of the task in the sample period. This is important because the proportion of time alone may be of limited interest unless related to some other quantity such as the frequency. For example, if the proportion of time on a particular task changes from one week to the next, it is impossible to tell without further information whether the change was due to a shift in the average time taken to complete the task, or simply to a change in the frequency of repetition of the task. In some cases it may be possible to relate a proportion to a quantity other than frequency, say, for example, to some measure of size of the overall operation. The availability of such a quantity will depend upon the specific application.

This paper discusses linear model estimation of the average time taken to perform specific tasks; the estimation is possible even when a number of different tasks are performed by an individual or group. The models are based on the conceptual relation that the time taken to perform a task multiplied by the frequency of its performance, and summed over all possible tasks, should equal the total time taken by all tasks. Estimation is also possible when direct observations are difficult to obtain (as in the stopwatch procedure) for the proposed models do not require direct time observations but rather utilize linear combinations of the individual time parameters.

Difficulties do arise, particularly in connection with the nonadditive manner in which people utilize their time; however, once obtained, the time estimates can be used for a number of purposes, such as, the construction of an efficiency index, the estimation of the size of a work force and the detection of strengths and weaknesses in the performance of particular tasks.

\section{(b) Cost Studies}

In cost analysis, estimates of components of a total cost are frequently of considerable interest. For example, if teams are sent to perform a number of different tasks, the costs associated with these different tasks may be unknown even though the total cost of the team operation may be precisely known. In fact in many cases, direct cost observations on the individual tasks may be virtually impossible to obtain. Again a conceptual relation is useful for model building, specifically, that the sum over all possible tasks, of the average cost of a task multiplied by its frequency, equals the total cost. The individual cost estimates could be used to project total costs and hence would seem to be particularly helpful in preparing contract proposals. 
Linear models can be applied to a wide variety of problems in which a total time or a total cost is to be broken into components. Since the example of Section III is a time study, Section II is discussed from the point of view of a time rather than a cost analysis. It should be remarked that while the time slice and stop watch procedures might be considered to be alternatives to the time application of the linear models No such alternatives seem to exist for cost analysis.

\section{The Linear Model Approach}

\section{(a) The Statistical Models}

In time studies, the linear model approach requires observations which consist of a total time and the frequencies with which various tasks are performed. Specifically, if the $i^{\text {th }}$ individual performs $k$ tasks with frequencies $f_{i 1}, f_{i 2}$, $\cdots, f_{i k}$ (some of which may be zero) and the total time spent by him performing all of these tasks is $t_{i}$, then the observations are of the form $\left(t_{i}, f_{i 1}, f_{i 2}, \cdots, f_{i k}\right)$, $i=1,2, \cdots, n$. In a given study these might include different workers in the same time period or the same workers in different time periods.

Using this notation, the ideal relationship described in Section I has the form

$$
t_{i}=\sum_{j=1}^{k} f_{i j} \alpha_{j} \quad i=1,2, \cdots, n
$$

where $\alpha_{j}$ is the time taken to perform task $j$. In practice however, human factors will ensure that such exact relationships do not hold. For example, all time will surely not be accounted for by the frequency with which specified tasks are performed. Nor will the time taken to carry out a task beadequately represented by a constant since it will clearly have variable properties associated with it. Thus one is lead to formulate the statistical model given in Equation (2.2).

Consider

$$
t_{i}=\sum_{j=1}^{k} f_{i j} w_{i j}+u_{i}
$$

$$
i=1,2, \cdots, n
$$

where

(i) $E\left(u_{i}\right)=0 E\left(u_{i}^{2}\right)=\tau^{2}, E\left(u_{i} u_{i^{\prime}}\right)=0, i \neq i^{\prime}$.

(ii) $w_{i j}$ is a random variable with expectation $\alpha_{j}$, i.e. $w_{i j}=\alpha_{j}+e_{i j}$, where: $E\left(e_{i j}\right)=0 ; E\left(e_{i j} e_{i^{\prime} j^{\prime}}\right)=0$ for $i \neq i^{\prime}$ and all $j$ and $j^{\prime}$; the $e_{i j^{\prime}}$ 's uncorrelated with the $u_{i}$ 's.

(iii) The covariance matrix of the random variable $w_{i}=\left(w_{i 1} \cdots w_{i k}\right)$ is given by the $k \times k$ matrix $\Sigma$.

When all $e_{i j}=0$ this formulation yields, as a special case, the ordinary model of linear regression.

The $u_{i}$ variables have been included to make up for the possible failure of the linear additive model to describe the operation precisely, and the $e_{i j}$ variables allow for variation in the time taken to perform a task. In some applications the variation in the $e_{i j}$ will be more important than the variation in the $u_{i}$. This will tend to be true if the work classifications successfully exhaust the uses of time, and if time is used in an additive way, for then terms are not "missing" from the model. Instances in which the $e_{i j}$ have been dominant and applications in which the $u_{i}$ clearly dominate have both been encountered. Each problem has to be examined for its unique characteristics. 
It is informative to rewrite the model as

$$
t_{i}=\sum_{j=1}^{k} f_{i j} \alpha_{j}+\gamma_{i},
$$

where

$$
\gamma_{i}=\sum_{j=1}^{k} f_{i j} e_{i j}+u_{i},
$$

so that $E \gamma_{i}=0$, and, in terms of the usual matrix multiplication,

$$
E \gamma_{i}{ }^{2}=\sigma_{i}{ }^{2}=F_{i} \Sigma F^{\prime}{ }_{i}+\tau^{2}, \quad F_{i}=\left(f_{i 1}, f_{i 2}, \cdots, f_{i k}\right)
$$

and

$$
E\left(\gamma_{i} \gamma_{l}\right)=0,
$$

This formulation is similar to the standard components of variance model. The differences are that the variance components model assumes that: (i) $\alpha_{j}=0$ for all $j$, (ii) the $w_{i j}$ are uncorrelated for each $i$, and (iii) that the variance components of $\Sigma$ are to be estimated.

\section{(b) Estimation}

The method of least squares is used to estimate the coefficients. However, certain difficulties arise; for example, when $\Sigma$ is unknown and is estimated from previous data, the distributional properties of the estimates may not be simple. Furthermore, the estimation of $\Sigma$ directly from the data of the study itself requires iterative minimization techniques.

Estimates can be found by assuming that $\Sigma=\Lambda \tau^{2}$ with $\Lambda$ known, giving $\sigma_{i}{ }^{2}=\left(F_{i} \Lambda F^{\prime}{ }_{i}+1\right) \tau^{2}=d_{i}{ }^{2} \tau^{2}$ with $d_{i}$ known. Then minimization of

$$
\sum_{i=1}^{n}\left[\left(t_{i}-\sum_{j=1}^{k} f_{i j} \alpha_{j}\right) / d_{i}\right]^{2}
$$

gives

$$
\hat{\alpha}=\left[(D F)^{\prime}(D F)\right]^{-1}(D F)^{\prime} D t
$$

where $D$ is $\operatorname{diag}\left(d_{1}^{-1}, d_{2}^{-1}, \cdots, d_{n}^{-1}\right), F^{\prime}=\left(F_{1}, F_{2}, \cdots, F_{n}\right)$, and $t^{\prime}=$ $\left(t_{1}, t_{2}, \cdots, t_{n}\right)$. The covariance matrix of $\hat{\alpha}$ is given by

$$
\left[(D F)^{\prime}(D F)\right]^{-1} \tau^{2}
$$

and the estimate of $\tau^{2}$ by

$$
\hat{\tau}^{2}=\frac{1}{n-k} \sum_{i=1}^{n}\left(\frac{t_{i}-\Sigma f_{i j} \hat{\alpha}_{j}}{d_{i}}\right)^{2}
$$

A useful special case occurs when $\Lambda=I$, so that $d_{i}{ }^{2}=\sum_{j=1}^{k} f_{i j}^{2}+1, d_{i}{ }^{2} \doteq$ $\sum_{j=1}^{k} f_{i j}^{2}$ and

$$
\hat{\alpha}=\left(C^{\prime} C\right)^{-1} C^{\prime} Z
$$

where

$$
c_{i j}=\frac{f_{i j}}{\sqrt{\sum_{j} f_{i j}^{2}}} \text { and } z_{i}=\frac{t_{i}}{\sqrt{\sum_{j} f_{i j}^{2}}}
$$


If, in addition to the diagonal covariance matrix, it is assumed that the variances of the $w$ 's are proportional to their expectations, and that the frequency of a task is in inverse proportion to the time that it takes to perform the task, then the weighting is by $\left(\Sigma f_{i j}\right)^{1 / 2}$ rather than by $\left(\Sigma f_{i j}^{2}\right)^{1 / 2}$.

\section{(c) Discussion of the Model}

(1) In the previous subsection the simplest model was obtained with the assumption of a diagonal covariance matrix, $\Sigma$. If the performance times of the $k$ work items represented in the matrix are thought to be correlated to the extent that a more general form of $\Sigma$ is being considered, then additional care might have to be taken in the formation of the model. For example, if performance times have a correlation induced by the time of office closing, then time of day might have to appear explicitly in the model. Or, if there is a strong natural correlation between two performance times then dropping one of the classifications might be considered.

It is also possible to introduce a correlated structure into the $u_{i}$ variables. No new problems are introduced by this assumption.

(2) It may not always be possible to describe the operation by a simple linear model without interactions. The performance time of a single work classification may depend on both its own frequency and the frequency with which other work items are performed. In the model representation, the addition of terms of the form $f_{i} f_{l} \alpha_{i l}$ may improve the precision of the fit and at the same time yield information about the operation itself. Interpretation of such interaction terms may not be at all simple, and, the existence of them would require that a very careful examination of the whole operation be made.

(3) The model specification, $w_{i j}=\alpha_{j}+e_{i j}$ implies that the $i^{\text {th }}$ person performing the $j^{\text {th }}$ task is above or below the average by the same amount each time the job is performed in the specified observation period. For this reason, it may sometimes be desirable to include in the model, a term, $e_{i j l}$, which depends on the person, the task, and the specific occurrence of the task, i.e. $w_{i j l}=\alpha_{j}+e_{i j}+$ $e_{i j l}$. No new problems are introduced by this assumption.

(4) In many cases, it will be informative to include a constant term in the linear model. This term may sometimes be interpreted as an estimate of overhead or idle time.

(5) In these problems, interest is focused on the coefficients which determine the relationship, and not simply on prediction of the independent variable. Cost studies, for example, might require both estimates of individual costs and of total cost. Similarly, time studies will often involve both individual time estimates and prediction of total time required; the latter for the scheduling of the size of work forces. Thus, the problem takes on some aspects of linear model prediction theory and some of the structural equations theory as used in econometrics.

\section{(d) Pooling}

It is entirely possible that exact or approximate linear relationships exist among the observed frequencies. These relationships may be known a priori, 
or, may simply appear in the data, but, in either case, they imply singularities in the normal equations and difficulties in estimation. The statistician is consequently in a difficult position because the usual statistical technique is to pool the regressors to eliminate the linear correlations. This however means that estimates will not be obtained for all work classifications. Unfortunately, in some cases, no satisfactory alternative will exist and pooling may be an unavoidable next step. Furthermore, the procedure will lead to another difficulty, namely that the pooling of correlated items to obtain a statistically satisfactory set of coefficients, does not necessarily yield a meaningful set, and so, in these applications, any pooling must be influenced by both statistical considerations and the meaning of the resultant coefficients. This is sometimes difficult to achieve.

It is important to notice that correlated frequencies cannot always be dropped from the model because interpretation of the coefficients requires that the frequency observations be exhaustive with respect to the time observation. In

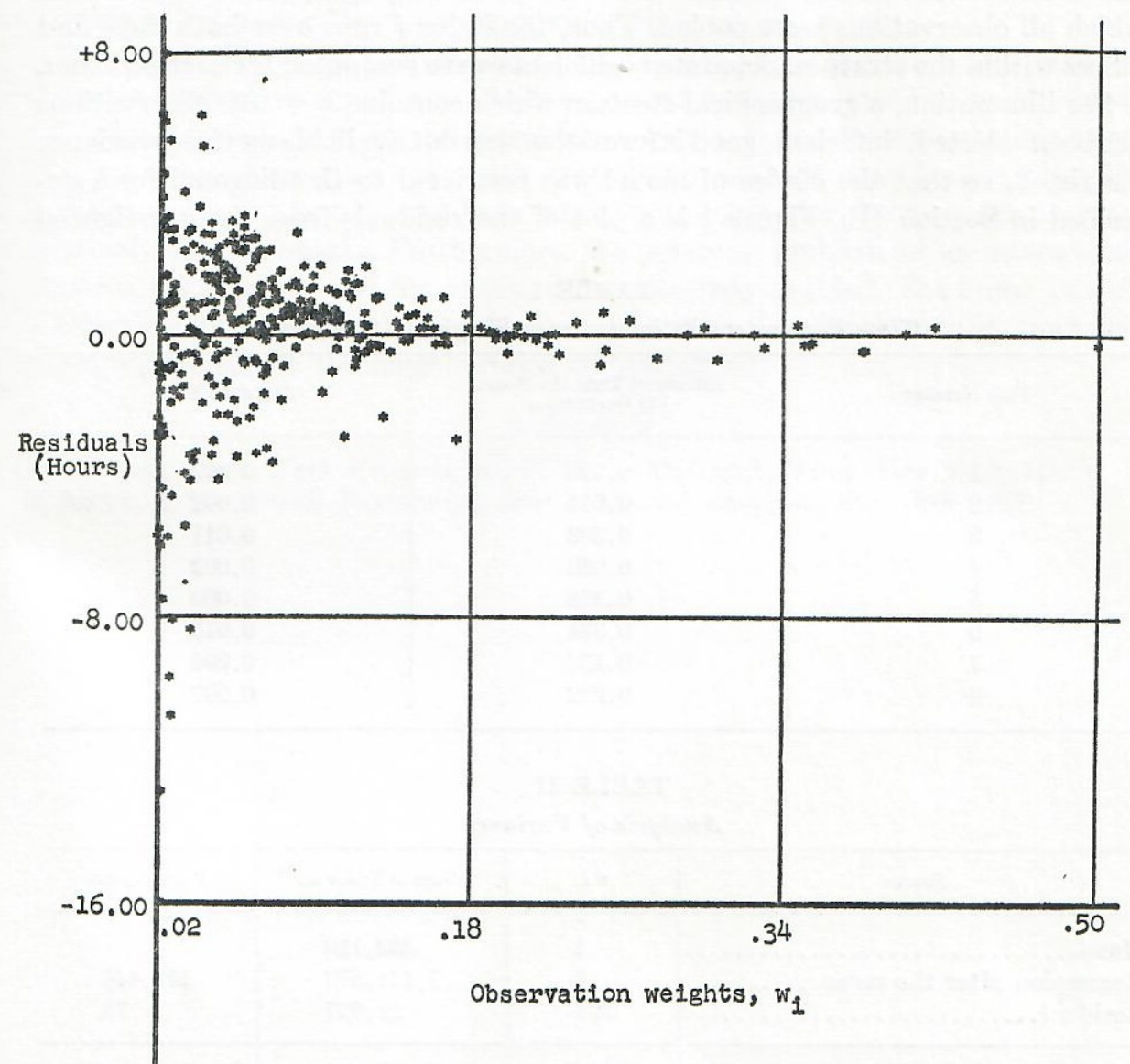

FIG. 1 
some studies enough information may be available to adjust the time observations to allow some frequencies to be omitted.

\section{A Specific Application}

In 1961 a study was made of the Business Offices in the Bell System. Observations were taken on each of five separate days in a number of different offices. These observations pertain to individuals working in groups in which any individual could be called upon to perform any or all of twelve different classifications of work.

The daily time observation, $t_{i}$, consists of the total work time put in by all individuals in the group during that day. Similarly, the frequency observation, $f_{i j}$, is the daily total over all individuals for the $j$ th work classification. Within each office approximately the same number of employees appeared in the study on all days. Thus an observation is available for each office for each of five days of the form $\left(t_{i}, f_{i 1}, f_{i 2}, \cdots, f_{i, 12}\right)$ where the $t_{i}$ and $f_{i j}$ are office totals. These offices were subsequently grouped into five different geographical strata within which all observations were pooled. Thus, the index $i$ runs over both days and offices within the stratum. Separate coefficients were computed for each stratum.

For illustration, a geographical stratum which contains $n=310$ observations has been selected. Sufficient, good information was not available on the covariance matrix, $\Sigma$, so that the choice of model was restricted to the diagonal form described in Section IIb. Figure 1 is a plot of the residuals from the unweighted

TABLE 1

\begin{tabular}{c|c|c}
\multicolumn{2}{c}{ Time Parameters Estimated with Weighting $\left(\Sigma f_{i j}^{2}\right)^{-1 / 2}$} \\
\hline Task Number & $\begin{array}{c}\text { Estimated Time (In Hours) } \\
\text { Per Occurrence }\end{array}$ & Standard Error \\
\hline 1 & 0.358 & 0.007 \\
2 & 0.014 & 0.002 \\
3 & 0.303 & 0.011 \\
4 & 0.010 & 0.002 \\
5 & 0.368 & 0.009 \\
6 & 0.334 & 0.015 \\
7 & 0.134 & 0.005 \\
8 & 0.222 & 0.007 \\
\hline
\end{tabular}

TABLE II

Analysis of Variance

\begin{tabular}{|c|c|c|c|}
\hline Source & d.f. & Sum of Squares & Mean Square \\
\hline $\begin{array}{l}\text { Mean } \ldots \ldots \ldots \ldots \ldots \ldots \ldots \\
\text { Regression after the mean } \ldots \ldots \ldots \ldots \ldots \\
\text { Residual } \ldots \ldots \ldots \ldots \ldots \ldots \ldots \ldots \ldots \ldots\end{array}$ & $\begin{array}{r}1 \\
8 \\
301\end{array}$ & $\begin{array}{r}352,110 \\
1,131,570 \\
21,967\end{array}$ & $\begin{array}{r}141,446 \\
73\end{array}$ \\
\hline Total. & 310 & $1,505,647$ & \\
\hline
\end{tabular}


regression against $w_{i}=d_{i}^{-1}$, and, clearly shows the inverse relationship which leads to the weighting of the observations by the sum of squares. In Table I the estimated times computed with weights $\left(\Sigma f_{i j}^{2}\right)^{-1 / 2}$ are presented with their standard errors. A summary of the statistical properties of the regression fit is presented in analysis of variance form in Table II. High correlations among certain frequencies necessitated pooling the work classifications down from twelve to eight, and, further, the customer service nature of the job required the inclusion of a constant term in the model. Consequently, Table II shows one degree of freedom for the mean and eight for the regression.

In summary, the linear model approach was used to estimate the average time required for eight work operations. The fact that the eight classifications resulted in a pooling from twelve original classifications was not regarded as a disadvantage because of a natural relationship among the pooled categories. For example, if an order form is filled out after each telephone call, it seems entirely satisfactory to consider the time on the telephone and the time required to fill out an order form together. Indeed in a case like this it may be physically impossible to determine the time required for each job separately.

The estimation of the time coefficients by means of linear models also allowed the estimation of overhead time. As was pointed out earlier this can be obtained by including a constant in the regression analysis. It is doubtful whether time slice work sampling could give such an estimate without considerable bias. In addition, sampling would have been much more costly, for the frequencies were relatively easy to obtain. Furthermore, the potential problem of an interaction between the observer and the employees was entirely avoided. The linear model without interactions fits well with the coefficients of variation of the time estimates generally in the neighborhood of two percent.

\section{References}

1. Abruzzi, Adam, Work Measurement, Columbia University Press, New York, 1952.

2. KRIcK, E. V., Methods Engineering, John Wiley and Sons, Inc., New York, 1962. 